\title{
Sensitivity enhancement by increasing the nonlinear crystal length in second-order autocorrelators for ultrashort laser pulses measurement
}

\author{
Sebastián Jarabo ${ }^{(\mathrm{a})}$, Enrique Rodríguez-Martín ${ }^{(\mathrm{b})}$, \\ José E. Saldaña-Díaz ${ }^{(a, c)}$, and Francisco J. Salgado-Remacha ${ }^{(a)}$
}

(a) Departamento de Física Aplicada, Universidad de Zaragoza, Pedro Cerbuna, 12, E-50009 Zaragoza, Spain.

(b) Fagor Automation, Barrio San Andrés, 19, E-20500 Arrasate-Mondragón, Spain.

(c) Facultad de Ciencias e Ingeniería, Departamento Académico de Ciencias Básicas, Universidad Nacional de Huancavelica, Huancavelica, Peru.

\section{Corresponding author}

Sebastián Jarabo, Departamento de Física Aplicada, Universidad de Zaragoza, Pedro Cerbuna, 12, E-50009 Zaragoza, Spain, e-mail: sjarabo@unizar.es, Phone: 349767610 00, Fax: 34976761233 .

\begin{abstract}
A theoretical model for interferometric autocorrelation with long nonlinear crystal (input depleted) has been developed and applied to the measurement of the duration of ultrashort pulses. The phase-matching condition is assumed throughout pulse spectrum. The interferometric autocorrelation trace of a mode-locked fibre laser $(20 \mathrm{~nJ}$ energy, $100 \mathrm{~kW}$ peak power, centred at $1595 \mathrm{~nm}$ ) has been measured by employing a fibre interferometer to avoid misalignment effects and a BBO nonlinear crystal as long as $2 \mathrm{~mm}$, in order to generate higher second-harmonic power. BBO crystal was used because it can keep the phasematching condition throughout a wide spectrum around $1600 \mathrm{~nm}$. By fitting the experimental measurements and computing according to the theoretical model exposed, it has been demonstrated that the autocorrelator sensitivity is clearly enhanced by increasing the nonlinear crystal length. A temporal duration of 0.18 ps has been obtained by fitting theoretical and experimental values.
\end{abstract}

\section{Keywords}

Pulse measurement; Interferometric autocorrelation; Second-harmonic generation; Phase matching; Mode-locked fibre laser 


\section{Introduction}

Accurate measure methods of ultrashort laser pulses in the time domain are essential tools in the development of new laser sources with shorter pulses, in the understanding of light-matter experiments and applications, in the advancement of our knowledge about pulsed lasers, etc. Although both energy and duration describe the main features of the pulse, the second one deserves more attention because the energy of each pulse can be easily raised by optical amplifiers, whereas more complicated techniques are necessary to shorten its temporal width.

There are several methods that can be employed to measure the duration of laser pulses [1]: intensity autocorrelation (non-collinear scheme), interferometric autocorrelation (collinear scheme), frequency-resolved optical gating applied to second-harmonic generation ("SHG FROG" technique) and other similar methods. All of them are mainly based on the second-harmonic generation by means of a nonlinear crystal (KTP, BBO, etc.) and, therefore, the pulse should have a high peak power. This limitation could be very restrictive in order to measure an only pulse by single-shot autocorrelation techniques.

Nevertheless it is also possible to measure low-power pulses provided that the pulsed laser emits a high-repetition-rate train of pulses, since the mean value of the second-harmonic power will be appreciable. Measurements of very weak pulses have been recently reported for different kinds of mode-locked lasers by means of intensity autocorrelation [2-5], interferometric autocorrelation [6-8] and SHG FROG [9-11]. Typically, the reported pulses have energies lower than $1 \mathrm{~nJ}$, although peak powers exceed $4 \mathrm{~kW}$ because their temporal widths vary from a few tens to a few hundreds of femtoseconds. It is important to notice that all of these lasers emit trains of pulses with a very high modulation frequency around 100 $\mathrm{MHz}$, typically. Thus, enhancements in experimental techniques are always useful to measure the duration of low-power pulses with low repetition rates or even to measure the duration of individual pulses (single-shot techniques).

These improvements can be obtained by enhancing detection sensitivity, but also by means of lengthening of the nonlinear crystal in order to increase the second-harmonic power. Usually, very thin crystals (15 $\mu \mathrm{m}$ [12]) are employed to keep the phase-matching broadband. Thus, as the input power is not depleted, the interferometric autocorrelation trace will have a contrast 1:8. However, this condition is not mandatory and if a suitable theoretical model were applied, it would be possible to work with longer crystals. Nevertheless, the nonlinear crystal must be suitably selected to keep the phase matching condition throughout the pulse spectrum.

In this paper the interferometric autocorrelation of a train of pulses emitted by a modelocked erbium-doped fibre laser (1595 nm, $20 \mathrm{~nJ}$ energy, $100 \mathrm{~kW}$ peak power [13]) is measured. A custom-made fibre interferometer (Michelson type) is employed in order to suppress misalignment effects and a $\mathrm{BBO}$ (Beta Barium Borate, $\beta-\mathrm{BaB}_{2} \mathrm{O}_{4}$ ) crystal is selected to keep the phase-matching broadband along the nonlinear crystal ( $2 \mathrm{~mm}$ length), since its phase-matching angle (PMA) is practically constant around $1600 \mathrm{~nm}$ (as opposed to $800 \mathrm{~nm}$ ). A duration of $0.18 \mathrm{ps}$ is obtained by means of fitting the autocorrelation trace to a suitable theoretical model, which is developed by assuming conditions of input depleted and of phase matching.

Thus, we demonstrate in this work that the sensitivity of an autocorrelator can be clearly improved by using a long nonlinear crystal. In our particular case, by considering the energy and the duration of each pulse aforementioned, our autocorrelator would need a sensitivity, 
defined by $\mathrm{S}=\mathrm{P}_{\text {mean }} \mathrm{P}_{\text {peak }}$, lower than $2 \times 10^{-3} \mathrm{~W}^{2}$ in order to obtain a right measurement of the duration of our pulses. By including a $2 \mathrm{~mm}$ length BBO crystal in our autocorrelator, its sensitivity reaches $10^{-5} \mathrm{~W}^{2}$ and therefore our pulses can be measured. However, the sensitivity increases as the length of the nonlinear crystal diminishes. In fact, if the length is shorter than $125 \mu \mathrm{m}$, the sensitivity is higher than $2 \times 10^{-3} \mathrm{~W}^{2}$ and it is not possible to measure the pulsewidth. In conclusion, the long length of our BBO crystal is a key feature, since the energy of our pulses is too low to employ very thin nonlinear crystals, as it is usually done in standard autocorrelators. It is necessary to point out that our autocorrelator has been specially implemented in order to demonstrate that the measurement sensitivity is improved by several orders of magnitude by means of increasing the length of the nonlinear crystal. Although it does not hope to compete against commercial autocorrelators (in our operating conditions, basic models of APE-Angewandte Physik \& Elektronik GmbH, Minioptic Technology Inc. or Femtochrome Research Inc. would provide sensitivities around $10^{-6} \mathrm{~W}^{2}$ ), the proposed method can be applied within any second-order autocorrelator to enhance its sensitivity, with clear advantages in measurements of very low energy pulses or in single-shot techniques.

\section{Theoretical description}

The temporal width of ultrashort laser pulses is usually measured by means of an alloptical method based on the autocorrelation function [1]. To measure this function, the common procedure consists in splitting the pulse under measurement into two replicas by means of an interferometer (Michelson, Mach-Zehnder, etc.), which adds a delay in one of them. Later, both replicas travel through a nonlinear crystal (BBO, KTP, etc.) and they generate power in the second harmonic. Finally, the second harmonic power is filtered and detected by a slow photodetector, whose response will depend on the delay added by the interferometer.

If a depleted input is considered, the second-harmonic intensity $I^{(2 \omega)}$ generated can be expressed [14] in the form

$$
I^{(2 \omega)}=I^{(\omega)} \tanh ^{2}\left[\zeta L \sqrt{I^{(\omega)}}\right]
$$

being $I^{(\omega)}$ the input intensity, $L$ the length of the nonlinear crystal and $\zeta$ a parameter that depends on the type of crystal and on the condition of phase-matching. On the other hand, if the instantaneous electromagnetic field $E(t)$ of the pulse is known, then the field in the input of the nonlinear crystal will be proportional to $E(t)+E(t-\tau)$, where $\tau$ is the delay adds by the interferometer. Therefore, the response of the photodetector can be expressed as follows

$$
V(\tau) \sim \int_{-\infty}^{+\infty} d t|E(t)+E(t-\tau)|^{2} \tanh ^{2}[\beta|E(t)+E(t-\tau)|]
$$

where the second-harmonic generation depends on the dimensionless parameter $\beta$, and $E(t)$ is considered dimensionless for convenience sake. This function is usually normalized as

$$
G(\tau)=\frac{V(\tau)}{V(\tau \rightarrow \infty)} ; G(\tau \rightarrow \infty)=1
$$


If $\tau \rightarrow \infty$, both pulses are not overlapped and the response must be twice the response due to a single pulse and therefore

$$
V(\tau \rightarrow \infty) \sim 2 \int_{-\infty}^{+\infty} d t|E(t)|^{2} \tanh ^{2}[\beta|E(t)|]
$$

and substituting in Eq. (3) is obtained that

$$
G(\tau)=\frac{1}{2} \frac{\int_{-\infty}^{+\infty} d t|E(t)+E(t-\tau)|^{2} \tanh ^{2}[\beta|E(t)+E(t-\tau)|]}{\int_{-\infty}^{+\infty} d t|E(t)|^{2} \tanh ^{2}[\beta|E(t)|]} .
$$

The normalized correlation is an even oscillating function, whose envelope tends asymptotically to the unity. Its values are comprised between 0 and a maximum value $G_{\max }$, which is only reached for $\tau=0$. Therefore, $G_{\max }$ can be expressed in the form

$$
G_{\text {max }}=G(0)=2 \frac{\int_{-\infty}^{+\infty} d t|E(t)|^{2} \tanh ^{2}[2 \beta|E(t)|]}{\int_{-\infty}^{+\infty} d t|E(t)|^{2} \tanh ^{2}[\beta|E(t)|]}
$$

As $\beta$ increases, the $G_{\max }$ value diminishes. In consequence, the $G_{\max }$ value is limited between 2 and 8 . If $\beta \rightarrow 0$, then $G_{\max }=8$, $\operatorname{since} \tanh (x) \underset{x \rightarrow 0}{\longrightarrow} x$. If $\beta \rightarrow \infty$, then $G_{\max }=2$, since $\tanh (x) \underset{x \rightarrow \infty}{\longrightarrow} 1$, and $G(\tau)$ approaches to the first-order autocorrelation function, which can be measured with a photodetector placed in the output of the interferometer (without second-harmonic generation), that is to say,

$G(\tau) \rightarrow G_{1}(\tau)=\frac{1}{2} \frac{\int_{-\infty}^{+\infty} d t|E(t)+E(t-\tau)|^{2}}{\int_{-\infty}^{+\infty} d t|E(t)|^{2}}$.

In consequence it will only be possible to obtain information about the coherence length of the pulse. In order to obtain a contrast of $8: 1$ in the $G(\tau)$ function, the $\beta$ value must be very low. Thus, a very short interaction length should be arranged, by means of a very thin nonlinear crystal or by using noncollinear interferometric correlation. The two-photon process which takes place in a semiconductor detector has a very low efficiency $(\beta \ll)$ and it could also be used to reach this high contrast. In any case, as $\beta$ is very low, Eq. (5) can be approximated as

$G(\tau) \rightarrow G_{2}(\tau)=\frac{1}{2} \frac{\int_{-\infty}^{+\infty} d t|E(t)+E(t-\tau)|^{4}}{\int_{-\infty}^{+\infty} d t|E(t)|^{4}}$,

which is the second-order autocorrelation function usually employed [1]. However, if it were necessary to measure very weak pulses regarding the sensitivity of the autocorrelator, all of these methods provide a very lacking signal. In order to improve it, despite the contrast is lower, collinear method with a long nonlinear crystal results preferable, since a higher value of $\beta$ implies a higher signal at the photodetector. 
Moreover, as the instantaneous electromagnetic field $E(t)$ of the pulse must include a factor $\exp \left(i \omega_{0} t\right)$, being $\omega_{0}$ its optical frequency, then $|E(t)+E(t-\tau)|$ has a strong dependence on $\cos \left(\omega_{0} \tau\right)$ and therefore $\mathrm{G}(\tau)$ values exhibit an oscillating behaviour. In fact, very near of the origin $(\tau \sim 3 f s), \cos \left(\omega_{0} \tau\right) \cong-1$ and $\mathrm{G}(\tau)$ diminishes to 0 because then $E(t-\tau) \cong-E(t)$.

Henceforth, the term "full width" of a function (as temporal as spectral) will always refer to its width when it equals its maximum value divided by e. By assuming a pulse with a Gaussian shape, the behaviour of the autocorrelation functions can be realistically illustrated. If the pulse intensity has a full width $\Delta \tau_{p}$, its electromagnetic field can be described in the following form

$E(t)=\exp \left[-2\left(t / \Delta \tau_{p}\right)^{2}\right] \exp \left\{i\left[\omega_{0} t-2 \delta\left(t / \Delta \tau_{p}\right)^{2}\right]\right\}$

where $\delta$ is defined as

$\delta=\sqrt{\left(\Delta \tau_{p} / \Delta \tau_{p 0}\right)^{2}-1}$

being $\Delta \tau_{p 0}$ the original width of the pulse (that is to say, without dispersion). Thus, if the pulse is chirped due to the chromatic dispersion, then $\Delta \tau_{p} \neq \Delta \tau_{p 0}(\delta \neq 0)$. The more chirped the pulse, the higher the $\delta$ value.

In general, $G(\tau)$ values can be computed by substituting Eq. (9) and Eq. (10) in Eq. (5) and later by applying standard numerical models. Nevertheless, Eq. (5) can be algebraically solved for the two limit cases aforementioned, $G_{1}(\tau)$ (Eq. (7)) and $G_{2}(\tau)$ (Eq. (8)). Thus, the first-order autocorrelation function can be expressed as follows

$G_{1}(\tau)=1+\exp \left[-\left(\tau / \Delta \tau_{p 0}\right)^{2}\right] \cos \left(\omega_{0} \tau\right)$

This function does not depend on $\Delta \tau_{p}$ and therefore it is not useful to determine the actual width of the pulse. Nevertheless, $\Delta \tau_{p 0}$ can be determined by measuring the output of the interferometer (without second-harmonic generation) since its envelope has a full width $2 \Delta \tau_{p 0}$. The second-order autocorrelation function can be expressed in the form

$$
\begin{aligned}
& G_{2}(\tau)=1+2 \exp \left[-2\left(\tau / \Delta \tau_{p}\right)^{2}\right]+\exp \left[-2\left(\tau / \Delta \tau_{p 0}\right)^{2}\right] \cos \left(2 \omega_{0} \tau\right)+ \\
& +4 \exp \left[-\frac{1}{2}\left(\tau / \Delta \tau_{p 0}\right)^{2}\right] \exp \left[-\left(\tau / \Delta \tau_{p}\right)^{2}\right] \cos \left(\frac{\delta \tau^{2}}{\Delta \tau_{p}^{2}}\right) \cos \left(\omega_{0} \tau\right)
\end{aligned}
$$

which provides information about the actual width of the pulse, since it depends on $\Delta \tau_{p}$.

In Fig. 1 autocorrelation function $G(\tau)$ computed by Eq. (5) by assuming Gaussian pulses is shown. Its qualitative behaviour is not affected by the $\beta$ value, but its contrast is clearly reduced as $\beta$ is increased. The maximum contrast (1:8) is found for $\beta=0$ and it diminishes to $1: 3$ for $\beta=1.25$. This change does not depend on the pulse width. Therefore, if $G(0)$ is known, then $\beta$ can be determined, since the dispersion have a negligible influence on $G(0)$. The relationship between both magnitudes is gathered in Fig. 2. As the secondharmonic generation raises, $G(0)$ diminishes and tends asymptotically to 2, that is to say, 
$G(0)$ tends to $G_{1}(0)$. Therefore, if $\beta$ reaches too high values, the autocorrelation function loses information about pulse dispersion. To avoid it, it is convenient that $\beta<2$.

Once that $\beta$ is known, the temporal width of the pulse can be obtained considering the half-width of the autocorrelation function, which is represented in Fig. 3 assuming a pulse without dispersion $(0.2 \mathrm{ps})$, since this condition is usually the most interesting. As it can be appreciated, the most suitable $\beta$ values are comprised between 1.25 and 1.5, approximately. In Fig. 3 the half-width of the autocorrelation function is also shown for chirped pulses with full widths of 0.4 ps and 1 ps.

\section{Experimental setup}

The experimental setup is arranged to measure the pulse duration of a $5.2 \mathrm{MHz}$ train of passive mode-locked pulses emitted by a ring-cavity erbium-doped fibre laser, which is reported in Ref. 13. It consists of a commercial erbium-doped fibre amplifier operating in the $\mathrm{C}$-band and a modulator based on the nonlinear polarization rotation effect. This modulator is formed by a linear polarizer placed between two polarization controllers. The spectrum of the pulses is centred at $1566 \mathrm{~nm}$ and its spectral width is around $35 \mathrm{~nm}$. Later, the laser pulses are amplified by a second commercial erbium-doped fibre amplifier operating in the L-band. The spectrum of the amplified pulses is shifted to $1595 \mathrm{~nm}$ and broadened up to $44 \mathrm{~nm}$. Although the energy of the amplified pulses is only around $20 \mathrm{~nJ}$, nonlinear effects are easily induced in highly nonlinear fibres, as it is reported in Ref. 15. Thus, it is expected that these pulses are very short, with a temporal duration lower than a few picoseconds.

In order to facilitate collinear autocorrelation and to avoid alignment problems, a Michelson interferometer with input and output by optical fibre has been employed. Its scheme is shown in Fig. 4. The input pulses are divided by means of a 50/50 coupler in the two arms of the interferometer. The optical paths of both arms are balanced by placing a fibre collimator and a flat mirror, which can be moved in a controlled way (Thorlabs servoactuator, model Z825B). The delay between both arms depends on the distance between the collimator and the mirror. If the mirror is displaced from the order-zero position, transmission losses are only increased by $0.07 \mathrm{~dB} / \mathrm{mm}$. However, transmission losses are practically unchanged since displacements during autocorrelation measurements are shorter than $0.3 \mathrm{~mm}$. The other arm employs an all-fibre mirror (50/50 coupler) and includes a variable optical attenuator and a polarization controller (General Photonics, model Polarite PLC-003-M-25) in order to equalize losses and polarization states between both arms. Transmission losses of the interferometer are $4.4 \mathrm{~dB}$ at $1600 \mathrm{~nm}$, slightly higher than expected losses of an ideal interferometer $(3 \mathrm{~dB})$, although the spectral variation of transmission losses is lower than $3 \%$ (over a spectral range of $44 \mathrm{~nm}$ ). Moreover, as it will be shown later, transmitted powers by both arms are correctly balanced, with a spectral variation lower than $4 \%$. Finally, it is necessary to point out that this interferometer has a weakness in comparison with other interferometers since air and silica optical fibre have a different chromatic dispersion. This handicap could be decisive to measure extremely short pulses.

To measure autocorrelation function, output pulses of the interferometer are overlapped along a $2 \mathrm{~mm}$ length BBO crystal, which generates second-harmonic power around $800 \mathrm{~nm}$. Residual power at $1600 \mathrm{~nm}$ is eliminated by an optical filter (Thorlabs, model FGB25). Although BBO is a nonlinear crystal with a large effective second-harmonic generation coefficient and a broad phase-matching range, it is necessary to check if the phase-matching condition is fulfilled over our spectral range (44 nm centred at $1595 \mathrm{~nm}$ ). Our crystal (Eksma Optics, model BBO-0608-16*, $\theta=19.9 \mathrm{deg}, \varphi=90 \mathrm{deg}$ ) is tailored to type I (ooe) generation 
at $1600 \mathrm{~nm}$. By applying second-harmonic generation theory [14] and considering ordinary and extraordinary refraction indexes determined by its Sellmeier coefficients, the phasematching angle in function of wavelength can be computed, as it is shown in Fig. 5. This angle only changes $0.1 \mathrm{deg}$ from $1550 \mathrm{~nm}$ to $1650 \mathrm{~nm}$. Therefore, the phase-matching factor, $\operatorname{abs}(\operatorname{sinc}(\Delta \mathrm{k} L / 2))[14]$, should be near unity along our spectral range. As it is seen in Fig. 5, for $\mathrm{L}=2 \mathrm{~mm}$, if $\theta=19.867 \mathrm{deg}$ (computed value at $1600 \mathrm{~nm}$ ), the spectral variation is lower than $1 \%$. Even if $\theta=19.9 \mathrm{deg}$ (specified value by Eksma Optics), the spectral variation is lower than 3\%. Obviously, by using shorter crystals, these values could be strongly diminished. In any case, these variations should not have significant influence on autocorrelation measurements.

\section{Results and discussion}

Interferometric autocorrelation traces are gathered in Figs. 6 and 7. In Fig. 6, the autocorrelation trace is measured by means of an InGaAs photodiode, but the nonlinear crystal is not placed in the experimental setup. Thus, it should correspond to the first-order autocorrelation function and by fitting to Eq. (11), the temporal width of the pulse without dispersion is found, being $\Delta \tau_{p 0}=0.17$ ps. On the other hand, Fig. 7 shows the measurement by employing the nonlinear crystal to generate second-harmonic power, which is detected by a Si photodiode. Now, the trace is due to the G correlation function and, by fitting to Eq. (5), the actual temporal width of the pulse $\Delta \tau_{p}$ is $0.18 \mathrm{ps}$ and the $\beta$-parameter value results 1.25 $(G(0)=3.1)$. As it was expected, this value is lower than 1 ps and the peak power of each pulse slightly exceeds $100 \mathrm{~kW}$. Although there are two fitting parameters, the procedure is simplified because they can be separately determined. First, $\beta$ parameter is obtained taking into account $\mathrm{G}(0)$. Next, the function envelope allows to fit $\Delta \tau_{p}$.

Measured and theoretical values are in good agreement. In fact, $\Delta \tau_{p 0}$ value corresponds with a gain-profile width of $31 \mathrm{~nm}$, in concordance with the spectral width of the pulse (35 $\mathrm{nm}$ ) emitted by the master laser. Therefore, although the second amplifier widens the spectral profile, it does not shorten the pulse duration. Nevertheless, it is necessary to point out that a Gaussian profile has been always assumed and, in consequence, the actual duration of the pulse could be slightly different.

It has been demonstrated that the experimental procedure is proper and that autocorrelation traces with/without BBO are clearly distinguishable. Moreover, both traces have not background in the origin (the two arms of the Michelson are well balanced), as it can be seen in Fig. 8. Although autocorrelation function has a contrast around 3:1 instead of 8:1, it is due to the length of the nonlinear crystal (second-harmonic generation with a depleted input), but it is possible to obtain right values for the pulse width whenever a suitable theoretical model is applied, as it has been exposed in Section 2.

Finally, once $\beta$ and $\Delta \tau_{\mathrm{p}}$ are known, it is possible to determine the sensitivity of the autocorrelator as a function of the nonlinear crystal length by means of Eq. (5) and considering the time response of the photodiode and the detection noise. As it is shown in Fig. 9, the autocorrelator sensitivity always improves as the crystal length is increased, although the sensitivity enhancement grows also with the modulation frequency of the train of pulses. By raising the crystal length from $10 \mu \mathrm{m}$ to $3 \mathrm{~mm}$, sensitivity values are diminished by 5 orders of magnitude if the modulation frequency is higher than $1 \mathrm{MHz}$. In Fig. 9 it can be also appreciated as the time width of our pulses can be rightly measured by including a $2 \mathrm{~mm}$ 
nonlinear crystal in the autocorrelator, but it could not be employed crystals shorter than 125 $\mu \mathrm{m}$.

\section{Conclusion}

In order to improve the measurement of the duration of pulses, it is possible to employ the interferometric autocorrelation method by means of long nonlinear crystals (higher second-harmonic power). Although the trace contrast is lower, the final result will be right provided that the trace is analysed by a suitable theoretical model, which must assume that the input is depleted. Thus, autocorrelation measurements can be fitted by means of two parameters, $\beta$ and $\Delta \tau_{p}$. The fitting procedure is not complicated, because both parameters are practically independent. Thus, $\beta$ is determined by the contrast of the interferometric trace and later $\Delta \tau_{p}$ is fitted by its envelope.

Nevertheless, it is necessary to choose carefully the type of nonlinear crystal, since the phase-matching condition must be kept along the crystal throughout pulse spectrum. In particular, BBO crystals are especially suitable for pulse wavelengths around 1500-1600 nm. In fact, a temporal duration of 0.18 ps have been measured for a train of pulses by means of a BBO crystal of $2 \mathrm{~mm}$ length and a good agreement have been found between theory (assuming input depleted) and experiment. By fitting the experimental measurements and by computing according to the theoretical model exposed, it has been demonstrated that the autocorrelator sensitivity is clearly diminished by increasing the nonlinear crystal length. This method can be applied in any second-order interferometric autocorrelator to improve its sensitivity. Finally, it is necessary to point out as a limitation of this work that a Gaussian profile has been always assumed and, in consequence, the actual duration of the pulse could be slightly different, although the conclusions should remain unaltered.

\section{Acknowledgements}

The authors wish to acknowledge Dr. Íñigo J. Sola for his valuable and helpful comments. This work was supported by the Ministerio de Economía y Competitividad (Programa Estatal de Fomento de la Investigación Científica y Técnica de Excelencia, project FIS2013-44174-P) and by the Diputación General de Aragón. J. E. Saldaña-Díaz acknowledges the financial support of Universidad de Zaragoza and Banco Santander (Doctoral Fellowship UZ-SANTANDER). 


\section{References}

1. L. Sarger, J. Oberlé, How to measure the characteristics of laser pulses. In: Rullière C, editor. Femtosecond Laser Pulses. Principles and Experiments, Springer-Verlag, Berlin, 1998, p. 195-222.

2. F. Pirzio, L. Fregnani,A. Volpi, A. Di Lieto, M. Tonelli, A. Agnesi, App. Opt. 55 (2016) 4414.

3. G. Sobon, J. Sotor, I. Pasternak, A. Krajewska, W. Strupinski, K.M. Abramski, Opt. Express 23 (2015) 31446.

4. J. Zhu, L. Wei, W. Tian, J. Liu, Z. Wang, L. Su, J. Xu, Laser Phys. Lett. 13 (2016) 055804.

5. D.A. Dvoretskiy, V.A. Lazarev, V.S. Voropaev, Z.N. Rodnova, S.G. Sazonkin, S.O. Leonov, A.B. Pnev, V.E. Karasik, A.A. Krylov, Opt. Express 23 (2015) 33295.

6. K. Krzempek, G. Sobon, P. Kaczmarek, K.M. Abramski, Laser Phys. Lett.10 (2013) 105103.

7. J. Ma, H. Huang, K. Ning, X. Xu, G. Xie, L. Qian, K.P. Loh, D. Tang, Opt. Lett. 41 (2016) 890.

8. Y. Tang, L.G. Wright, K. Charan, T. Wang, C. Xu, F.W. Wise, Optica 3 (2016) 948.

9. Y. Nomura, T. Fuji, Opt. Express 22 (2014) 12461.

10. X. Li, W. Zou, G. Yang, J. Chen, IEEE Photon. Technol. Lett. 27 (2015) 93.

11. M. Murtagh, J. Lin, R.P. Mildren, G. McConnell, D.J. Spence, Opt. Express 23 (2015) 15504.

12. J.K. Ranka, A.L. Gaeta, A. Baltuska, M.S. Pshenichnikov, D.A. Wiersma, Opt. Lett. 22 (1997) 1344.

13. C. Lavieja, S. Jarabo, M. Marín-Doñágueda, I.J. Sola, Opt. Fiber Technol. 19 (2013) 476.

14. A. Yariv, Second-harmonic generation and parametric oscillation. In: Optical electronics in modern communications, Oxford University Press; 1997, p. 273-323.

15. J.E. Saldaña-Díaz, S. Jarabo, F.J. Salgado-Remacha, Laser Phys. Lett. 13 (2016) 095012. 


\section{Figure captions}

Figure 1. Autocorrelation function $G(\tau)$ computed by Eq. (5) for Gaussian pulses with a full width of 0.2 ps (without dispersion) with different parameters: (a) 0.2 ps full width and $\beta=0$; (b) 0.4 ps full width (chirped pulse) and $\beta=0$; (c) 1 ps full width (chirped pulse) and $\beta=0$; (d) 0.2 ps full width and $\beta=1.25$; (e) 0.4 ps full width and $\beta=1.25$; (f) 1 ps full width and $\beta$ $=1.25$.

Figure 2. Maximum values of the autocorrelation function $G(0)$ in function of $\beta$ for a pulse duration of $0.2 \mathrm{ps}$. As the second-harmonic generation raises, $G(0)$ diminishes and tends asymptotically to 2 (grey line), that is to say, $G(0)$ tends to $G_{1}(0)$.

Figure 3. Autocorrelation function half-width in function of $\beta$ for a pulse with a full width of: 0.2 ps (without dispersion; red line), 0.4 ps (chirped pulse; green line), and 1 ps (more chirped pulse; blue line).

Figure 4. Scheme of the autocorrelator: $\mathrm{S}=$ servo-actuator; $\mathrm{M}=$ aluminium flat mirror; $\mathrm{C}=$ collimator (gradient-index lens); $\mathrm{PC}=$ polarization controller; $\mathrm{A}=$ variable attenuator; $\mathrm{L}=30$ $\mathrm{mm}$ focal length lens; $\mathrm{F}=$ bandpass filter $(715-1095 \mathrm{~nm})$; $\mathrm{BBO}=$ nonlinear crystal of $\mathrm{b}$ barium borate ( $2 \mathrm{~mm}$ length); $\mathrm{PD}=$ photodiode ( $\mathrm{Si}$ or InGaAs).

Figure 5. Phase-matching angle (PMA; grey line) and phase-matching factor as a function of the wavelength. BBO length is $2 \mathrm{~mm}$. To compute phase-matching factor, two angles have been considered: $\theta=19.867 \mathrm{deg}$ (computed value at $1600 \mathrm{~nm}$; dotted line) and $\theta=19.9 \mathrm{deg}$ (specified value by Eksma Optics; solid line). Dot-dashes lines delimit the spectral range of our pulses.

Figure 6. First-order autocorrelation trace (red line) and fitted theoretical function (green line). The temporal width of the Fourier-limited pulse is fitted to $0.17 \mathrm{ps}$.

Figure 7. Autocorrelation trace (red line) and fitted theoretical function (green line). The temporal width of the pulse is fitted to 0.18 ps. The $\beta$-parameter value results 1.25 . The contrast is around 3:1 instead of $8: 1$.

Figure 8. Detail of the autocorrelation traces measured: first-order autocorrelation (grey line) and $\mathrm{G}$ autocorrelation (black line).

Figure 9. Autocorrelator sensitivity in function of the nonlinear crystal length for several modulation frequencies. 

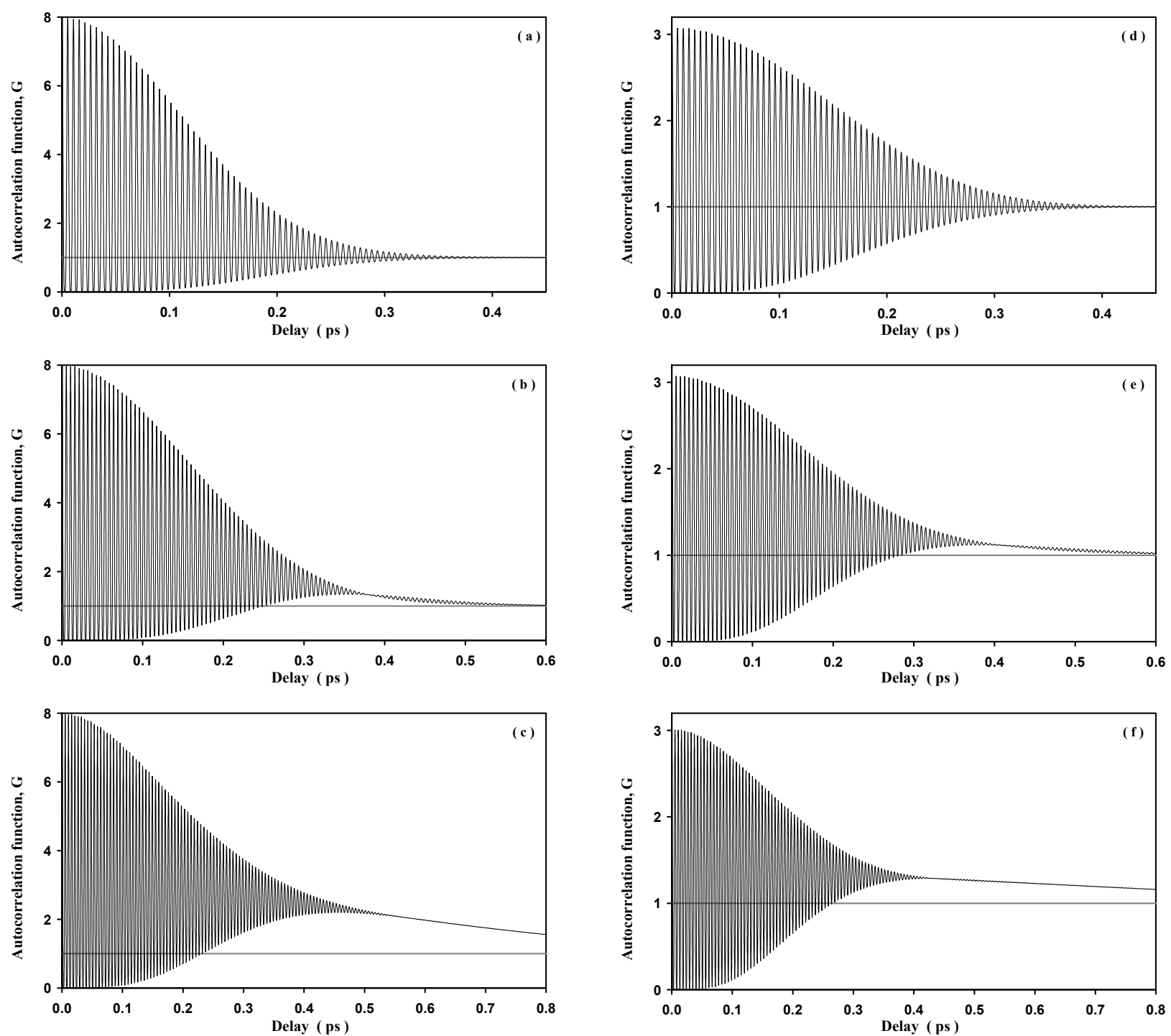

Fig 1. Autocorrelation function $G(\tau)$ computed by Eq. (5) for Gaussian pulses with a full width of $0.2 \mathrm{ps}$ (without dispersion) with different parameters: (a) 0.2 ps full width and $\beta=0$; (b) 0.4 ps full width (chirped pulse) and $\beta=0$; (c) 1 ps full width (chirped pulse) and $\beta=0$; (d) 0.2 ps full width and $\beta=1.25$; (e) $0.4 \mathrm{ps}$ full width and $\beta=1.25$; (f) 1 ps full width and $\beta=1.25$. 


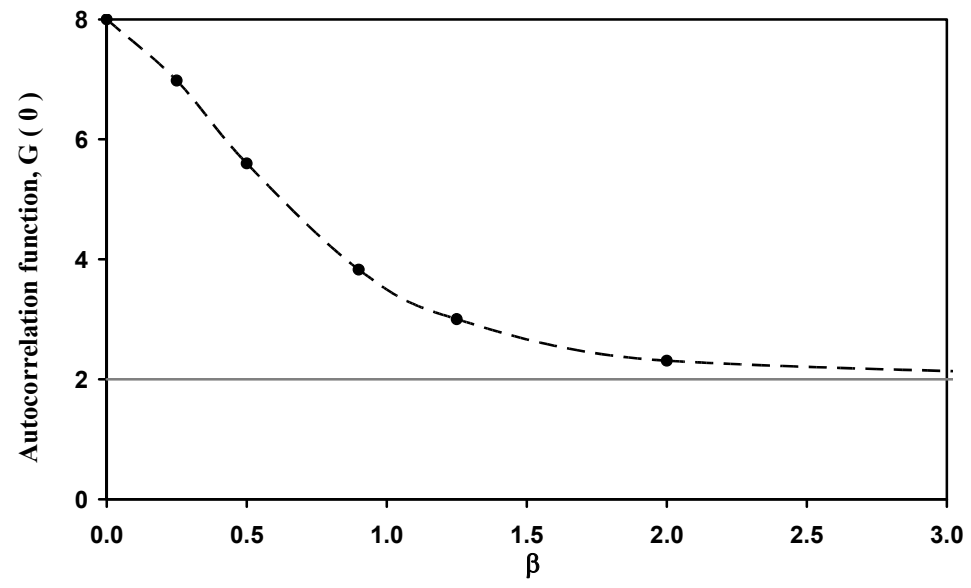

Fig 2. Maximum values of the autocorrelation function $G(0)$ in function of $\beta$ for a pulse duration of 0.2 ps. As the second-harmonic generation raises, $G(0)$ diminishes and tends asymptotically to 2 (grey line), that is to say, $G(0)$ tends to $G_{1}(0)$. 


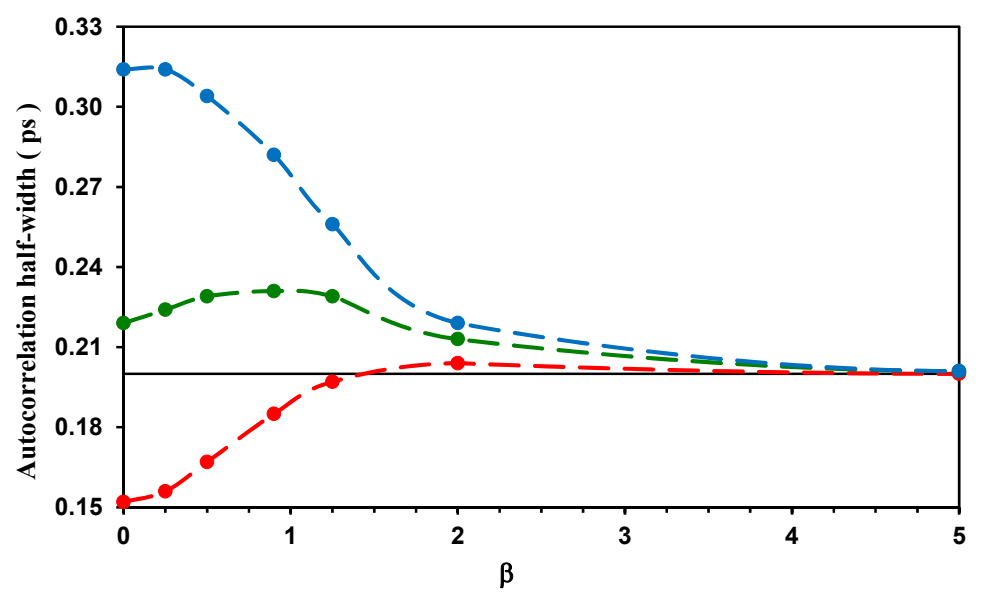

Fig 3. Autocorrelation function half-width in function of $\beta$ for a pulse with a full width of: $0.2 \mathrm{ps}$ (without dispersion; red line), $0.4 \mathrm{ps}$ (chirped pulse; green line), and $1 \mathrm{ps}$ (more chirped pulse; blue line). 


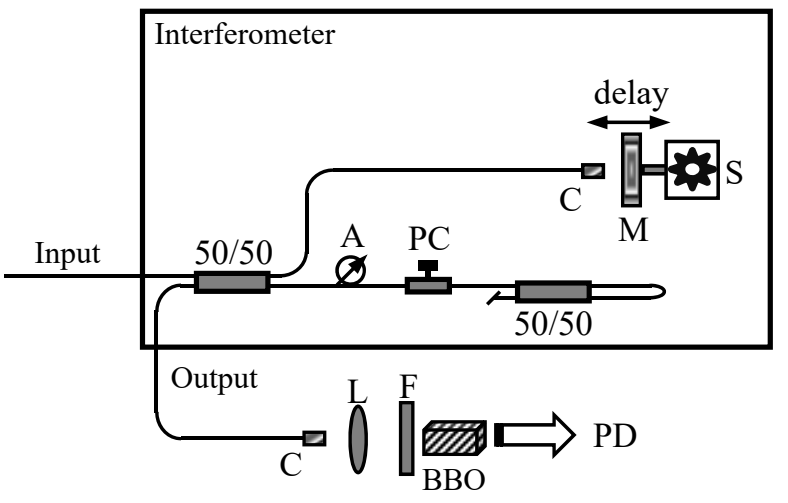

Fig 4. Scheme of the autocorrelator: $S=$ servo-actuator; $M=$ aluminium flat mirror; $\mathrm{C}=$ collimator (gradient-index lens); $\mathrm{PC}=$ polarization controller; $\mathrm{A}=$ variable attenuator; $\mathrm{L}=30 \mathrm{~mm}$ focal length lens; $\mathrm{F}=$ bandpass filter (715-1095 $\mathrm{nm}) ; \mathrm{BBO}=$ nonlinear crystal of $\beta$-barium borate $(2 \mathrm{~mm}$ length $) ; \mathrm{PD}=$ photodiode (Si or InGaAs). 


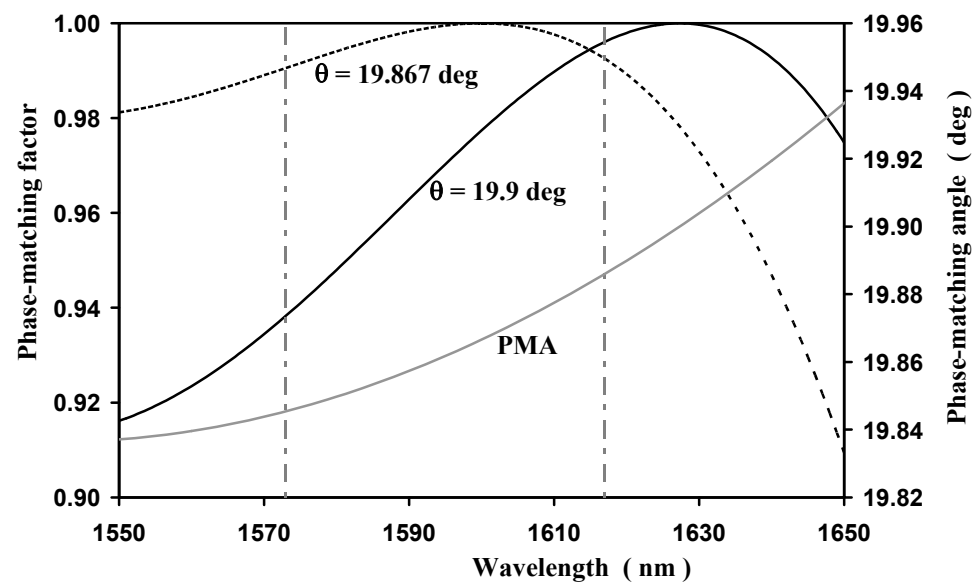

Fig 5. Phase-matching angle (PMA; grey line) and phase-matching factor as a function of the wavelength. BBO length is $2 \mathrm{~mm}$. To compute phase-matching factor, two angles have been considered: $\theta=19.867 \mathrm{deg}$ (computed value at 1600 $\mathrm{nm}$; dotted line) and $\theta=19.9 \mathrm{deg}$ (specified value by Eksma Optics; solid line). Dot-dashes lines delimit the spectral range of our pulses. 


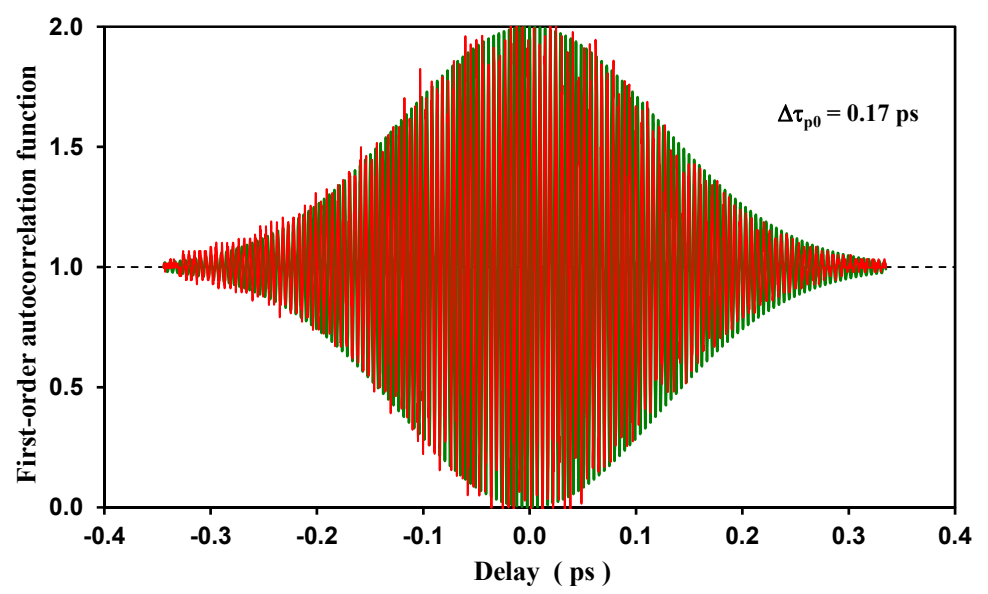

Fig 6. First-order autocorrelation trace (red line) and fitted theoretical function (green line). The temporal width of the Fourier-limited pulse is fitted to $0.17 \mathrm{ps}$. 


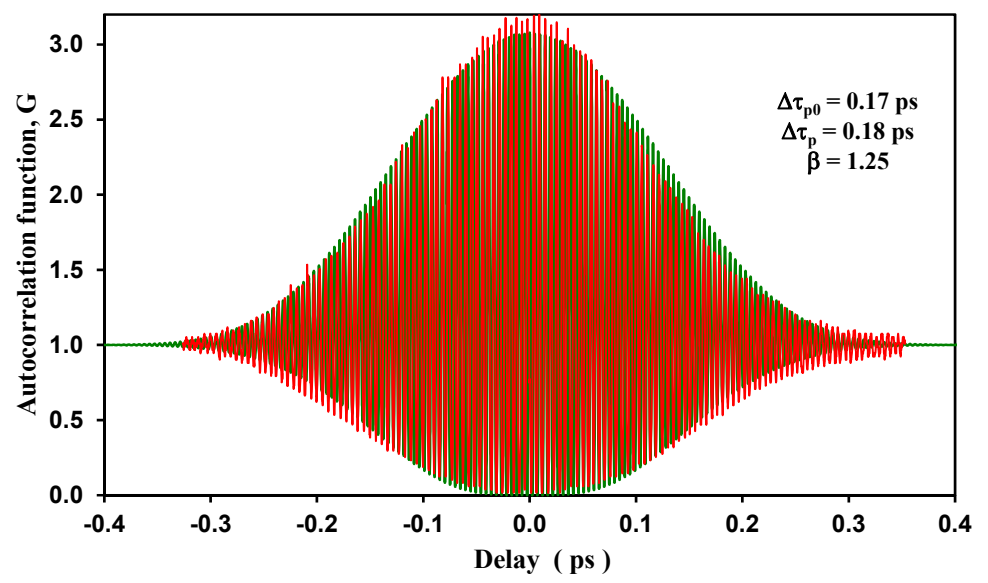

Fig 7. Autocorrelation trace (red line) and fitted theoretical function (green line). The temporal width of the pulse is fitted to $0.18 \mathrm{ps}$. The $\beta$-parameter value results 1.25. The contrast is around $3: 1$ instead of $8: 1$. 


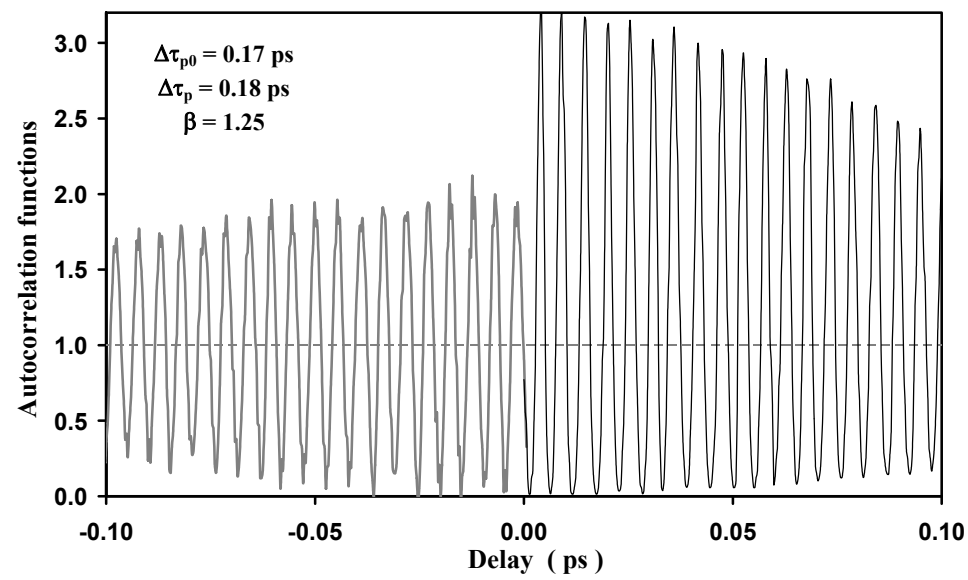

Fig 8. Detail of the autocorrelation traces measured: first-order autocorrelation (grey line) and $\mathrm{G}$ autocorrelation (black line). 


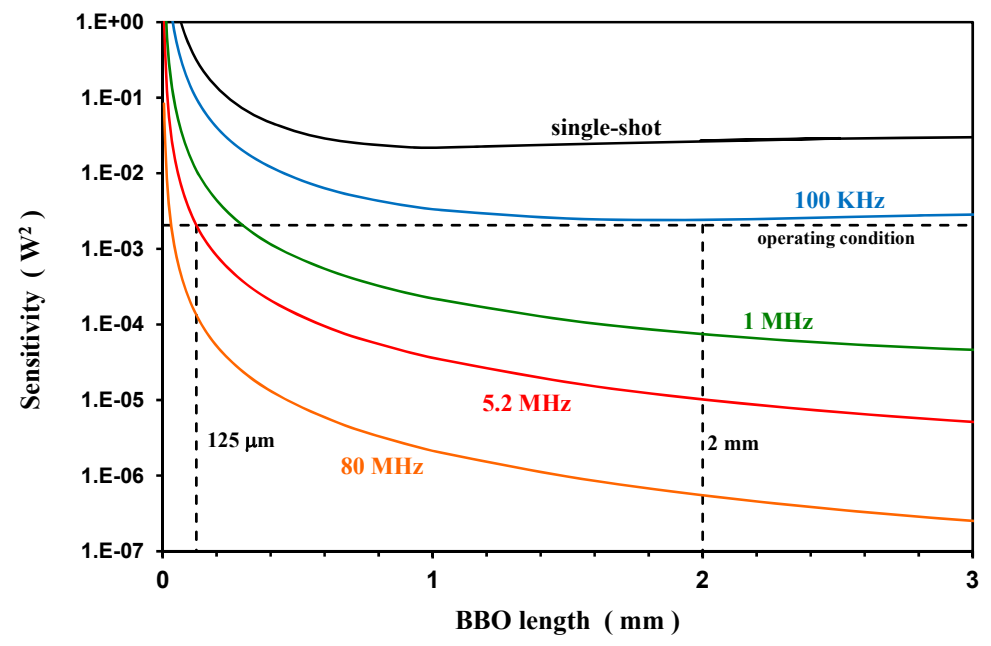

Fig 9. Autocorrelator sensitivity in function of the nonlinear crystal length for several modulation frequencies. 